\title{
Experience of the impact of physician associates on postgraduate medical training: A mixed methods exploratory study 滗
}

\author{
Authors: Sam Roberts, ${ }^{A}$ Sarah Howarth, ${ }^{B}$ Helen Millott ${ }^{C}$ and Laura Stroud ${ }^{D, E}$
}

The introduction of new professional roles to the multidisciplinary team has been heralded as a way to facilitate medical education in the face of increasing service pressures. However, concerns have been raised that the training of new healthcare professionals will dilute the availability of learning opportunities, thereby detracting from postgraduate medical education. As part of a mixed methods exploratory study, the experienced impact of newly qualified physician associates on medical training was explored. Within 6 months of newly qualified physician associates being introduced, half of junior doctors reported no overall impact on their training and $a$ third felt that their training was enhanced by the presence of physician associates. A minority of trainees experienced dilution of training opportunities. The findings support the notion that new healthcare professionals do not detract from medical training and suggest that roles such as the physician associate have potential to enhance postgraduate medical education.

KEYWORDS: Training, physician associate, postgraduate medical education

\section{Introduction}

Postgraduate medical education (PGME) is facing unprecedented pressure due to increasing service demands and conflicting priorities for training. ${ }^{1-3}$ Within this context, the NHS workforce continues to grow and diversify in an attempt to keep up with the healthcare needs of the UK population. ${ }^{4}$ Expansion in medical training has failed to keep pace with service demands and in order to create a sustainable future workforce, it is clear that many tasks previously considered the remit of doctors should, and are, being carried out by other healthcare professionals. 4,5

Physician associates (PAs), are well established in the USA but remain a new professional group in most areas of the UK with

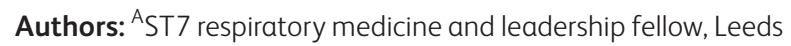
Institute of Health Sciences, Leeds, UK; ${ }^{\text {B }}$ research assistant, Leeds Institute of Medical Education, Leeds, UK; C ${ }^{\text {programme lead }}$ MSc PA studies, Leeds Institute of Medical Education, Leeds, UK; ${ }^{D}$ director, Leeds Institute of Health Sciences, Leeds, UK; ${ }^{E}$ associate dean, School of Medicine (Student Education), Leeds, UK an estimated 450 practicing PAs in $2017 .^{6}$ As such, their impact on UK PGME is unclear. ${ }^{7}$ Whereas roles such as advanced clinical practitioner (ACP) and pharmacist prescriber have emerged as a result of upskilling of existing healthcare professionals with their own regulatory bodies, training and continuing professional development structures, many aspects of the PA curriculum and post qualification educational needs overlap with medical training. ${ }^{8,9}$ As a result, concerns have been raised from several quarters that the presence of PAs will detract from postgraduate medical training. ${ }^{10-13}$ Specific concerns are that informal teaching opportunities, procedural skills training and funding for organised training programmes will be diverted to PAs. ${ }^{13-15}$ Whilst others have suggested that these concerns may be misplaced, citing international evidence, the impact of PAs on UK PGME is an area of intense debate with little published evidence upon which to base any discussion. ${ }^{14,16-19}$ With the numbers of practicing PAs set to increase exponentially over the coming years, information about their effect on medical education is urgently needed in order to plan the training of the NHS workforce of the future. ${ }^{7}$

As part of a wider exploratory study examining the impact and integration of newly qualified PAs (NQPAs) into the NHS workforce, we investigated experiences of the impact of PAs on PGME exploring the extent to which PAs facilitated and detracted from postgraduate medical training. ${ }^{20}$

\section{Methods}

This work was conducted as part of a mixed methods exploratory study undertaken in the north of England where the first cohort of locally trained PAs had just qualified and were being employed by five hospitals in the region. Wider study design was based on grounded theory with no a priori assumption of themes. ${ }^{21}$

\section{Participants}

All 22 PAs working or with provisional offers of employment in secondary care in the region were approached to take part. Twenty PAs were eventually employed across five different hospital sites from October 2017 onwards.

A number of junior doctors, consultants and ACPs in departments planning to employ PAs were also invited to take part, selected from a list of all staff within those departments. A purposive convenience sampling method was used to achieve maximum variation sampling across specialties and roles, aiming 
to invite 10 individuals and recruit three from each department planning to employ PAs. The number of participants recruited to each phase by role and specialty is shown in Table 1.

\section{Data Collection}

An iterative approach to data collection was adopted in keeping with the exploratory design with mixed methods used in a complementary manner to enrich data acquisition. ${ }^{22}$ Consequently, the study involved four distinct phases.

> Phase 1 - February to September 2017. Discussions took place with stakeholders to refine the wider study focus and inform development of the questionnaire for survey 1 . Stakeholders included junior doctors, senior clinicians, medical and PA educators, ACPs and experienced PAs.

> Phase 2 - September 2017. Survey 1 was distributed via email to 216 potential participants (19 PAs and 197 PA colleagues) prior to NQPAs starting work. Participants were asked to indicate their interest in participating in a future focus group. Staff expressing an interest but unable to attend focus groups were invited to semi-structured interviews.

> Phase 3 - December 2017 to February 2018. Focus groups and semi-structured interviews with PAs and colleagues (2-4 months after the NQPAs started work) took place. In addition to participants recruited via survey 1 , an email invitation was sent to all junior doctors working across the five hospitals, inviting them to attend focus groups.

> Phase 4-March to April 2018 (6 months after PAs started work). Survey 2 was distributed via email to all 20 PAs employed as well as all 167 doctors invited to survey 1 who had worked with PAs. ACPs were not invited to complete survey 2 as, in practice, very few worked directly with PAs. In addition, some departments had failed to recruit a PA therefore doctors working in these departments were not invited to survey 2 .

\section{Measures}

\section{Quantitative}

Survey 1 focussed on expectations of the impact of PAs including specific questions regarding participant access to training and expected impact of PAs on training. Most responses were answered on a five point Likert scale and free text items were included to capture additional concepts. Survey 2 focussed on experiences of working with PAs including the impact on postgraduate medical training, barriers to training and observed diversion of training opportunities to PAs. Both surveys were designed and disseminated using Jisc Online Surveys (Bristol, UK). All survey responses were submitted anonymously. Further information on survey questions are available in supplementary material S1.

\section{Qualitative}

Focus group and interview guides were developed from survey 1 responses and covered NQPA team integration, role overlap, impact on PGME, benefits and challenges of working with PAs. Focus groups and interviews lasted 20-60 minutes. These were audio-recorded and conducted by Sam Roberts (SR) with Sarah Howarth (SH) keeping field notes in larger focus groups. Focus groups/interviews continued until data saturation was achieved.
Table 1. Number of participants in each phase of

the study by specialty/role

\begin{tabular}{|c|c|c|c|c|}
\hline \multicolumn{2}{|c|}{ Participant Characteristics } & \multirow{2}{*}{$\begin{array}{l}\text { Phase } 2 \\
3\end{array}$} & \multirow{2}{*}{$\begin{array}{l}\text { Phase } 3 \\
11\end{array}$} & \multirow{2}{*}{$\begin{array}{l}\text { Phase } 4 \\
12\end{array}$} \\
\hline Specialty & Acute medicine & & & \\
\hline & Cardiology & 0 & 1 & 2 \\
\hline & $\begin{array}{l}\text { Colorectal/general } \\
\text { surgery }\end{array}$ & 3 & 3 & 5 \\
\hline & Dermatology & 1 & 3 & 0 \\
\hline & Elderly medicine & 2 & 5 & 6 \\
\hline & Gastroenterology & 1 & 1 & 1 \\
\hline & $\begin{array}{l}\text { Haematology/ } \\
\text { oncology }\end{array}$ & 3 & 1 & 7 \\
\hline & Paediatrics & 2 & 2 & 5 \\
\hline & Respiratory medicine & 5 & 2 & 10 \\
\hline & $\begin{array}{l}\text { Trauma and } \\
\text { orthopaedics }\end{array}$ & 1 & 2 & 8 \\
\hline & $\begin{array}{l}\text { Upper } \\
\text { gastrointestinal } \\
\text { surgery }\end{array}$ & 1 & 4 & 2 \\
\hline & Vascular surgery & 0 & 2 & 3 \\
\hline & Other & 8 & 0 & 5 \\
\hline \multirow[t]{6}{*}{ Role } & Consultant & 14 & 7 & 16 \\
\hline & Doctor ST3-ST8 & 2 & 2 & 15 \\
\hline & $\begin{array}{l}\text { Doctor CT1-2 (or } \\
\text { equivalent) }\end{array}$ & 3 & 4 & 8 \\
\hline & Foundation doctor & 4 & 5 & 19 \\
\hline & Physician associate & 11 & 16 & 10 \\
\hline & $\begin{array}{l}\text { Advanced clinical } \\
\text { practitioner (or } \\
\text { equivalent) }\end{array}$ & 2 & 2 & 0 \\
\hline Total & & 36 & 36 & 68 \\
\hline
\end{tabular}

\section{Data analysis}

\section{Quantitative}

Quantitative data from survey responses was collated and analysed using Microsoft Excel (97-2003). Descriptive statistics were used.

\section{Qualitative}

Thematic analysis was conducted on narrative data using the approach described by Braun and Clarke ${ }^{23}$ and organised using a qualitative data analysis tool (NVIVO Version 11, QSR International (UK) Ltd). Initial focus group recordings were analysed separately by two authors (Helen Millott (HM) and SR) following which a coding criterion was agreed. Coding of the remaining interviews was conducted by SR initially, following which themes were explored, compared and contrasted by three 
investigators (HM, SH and SR) until all were satisfied that the themes presented were reflective of participant experience.

Quantitative and qualitative data were then synthesised and interpreted together in the approach described by Plowright. ${ }^{22}$ Both quantitative and qualitative data are presented together with structure derived from thematic analysis. ${ }^{22,23}$

Quotes are tagged with the participant's role (foundation year 1 doctor (F1), foundation year 2 doctor (F2), core trainee doctor (CT), specialty trainee doctor (ST) or PA) followed by a numerical indicator or survey.

\section{Results}

At the start of the study, two of the five sites employed one PA each. Three employed no PAs. Eighteen NQPAs began working across the five sites between study phase 2 and phase 3 . The majority of NQPAs were ward based, rotating through different clinical areas every four months with around half also working out of hours in acute medicine.

Specialties employing PAs:

$>$ acute medicine

$>$ cardiology

$>$ colorectal surgery

$>$ dermatology

$>$ elderly medicine

$>$ emergency medicine

$>$ gastroenterology

$>$ haematology

$>$ paediatrics

$>$ respiratory medicine

$>$ trauma and orthopaedics

$>$ upper gastrointestinal surgery

$>$ vascular surgery.

There were 36 responses to survey 1 (response rate 17\%) and 68 responses to survey 2 (response rate $40.7 \%$ ). Data from survey 1 was used to inform study approach but the response rate was poor. As such, data presented here is derived only from phase 3 (focus groups and interviews) and phase 4 (survey 2) of the study unless explicitly stated. Participant roles are outlined in Table 1.

Thematic analysis revealed five key areas influencing the impact of PAs on PGME:

$>$ influence of workforce pressures on training

$>$ departmental culture

$>$ experience of working with PAs

$>$ concerns for future impact of PAs on training

$>$ training needs of PAs.

Full discussion of thematic analysis for the wider study is reported elsewhere. ${ }^{20}$ A full list of responses to questions relating to PGME in survey 2 are available in supplementary material S1.

\section{Influence of workforce pressures on training}

Workforce pressures were a key factor influencing access to training and the impact of the PA. Of the 42 junior doctors that responded to survey $2,38(90.5 \%)$ reported having missed training due to staff shortages within the last year and 22 (52\%) reported missing training at least monthly due to staff shortages; F1 (2):

I guess I don't feel like I'm in training a lot of the time. and CT (3):

One of the things that frustrated me about F1 was that you didn't get opportunities [to train] because there were so many gaps and because you were just overworked.

All participants were asked about the impact of the PA presence. $42(72 \%)$ felt that their presence reduced the impact of staff shortages and $46(79.3 \%)$ felt that they relieved pressure from other members of staff; ST (2):

They are quite useful on medical admission/assessment unit because they clerk patients and stuff and it takes pressure off.

\section{Departmental culture}

Departmental culture relating to education and training emerged from thematic analysis to strongly influence inter-professional collaboration and the impact of the PA role. Junior doctors reported a mean of 4.6 hours organised local training available per month. In some cases, junior doctors reported that they understood that PAs had been employed to enable them to attend training. This was interpreted as a positive step towards improving their training; F2 (1):

I think the reason why [sic] she was put on that particular ward was to give us time to go to training.

Where educational provision was limited, participants reported a sense of rivalry over training opportunities and a need to justify attendance at training; PA (13):

It's very difficult, because it's a very small team, to try and get to any training. If there is training organised for the physician associate cohort then you get away for that, but I'll often be questioned as to whether it's necessary.

Conversely, a positive departmental attitude to training was viewed by participants to contribute to inter-professional collaboration and sharing of training opportunities; PA (12):

There's quite a lot of training ... They have X-ray meetings and safety huddle things and the junior doctors teaching as well so sometimes I get invited to those and other times I'm the cover for them when they go which is fine because that's what we're supposed to do.

\section{Experience of working with PAs}

Participants' experiences of working with PAs was varied. Junior doctors were asked about whether the presence of a PA had influenced their attendance at organised teaching. Sixteen $(38.1 \%)$ reported they were more likely and three $(7.1 \%)$ reported that they were less likely to be able to attend as a result of working with a PA; ST (survey):

Extra bodies doing the tasks on the jobs list means freeing up trainees to learn, as they are not covering rota gaps.

Workplace-based training was also explored. Seven (16.7\%) junior doctors reported the presence of PAs reduced opportunities to complete workplace-based assessments. Focus group participants however reported no impact on availability of supervised learning events. The impact on the ability of junior doctors to undertake practical skills training was mixed. Whilst 


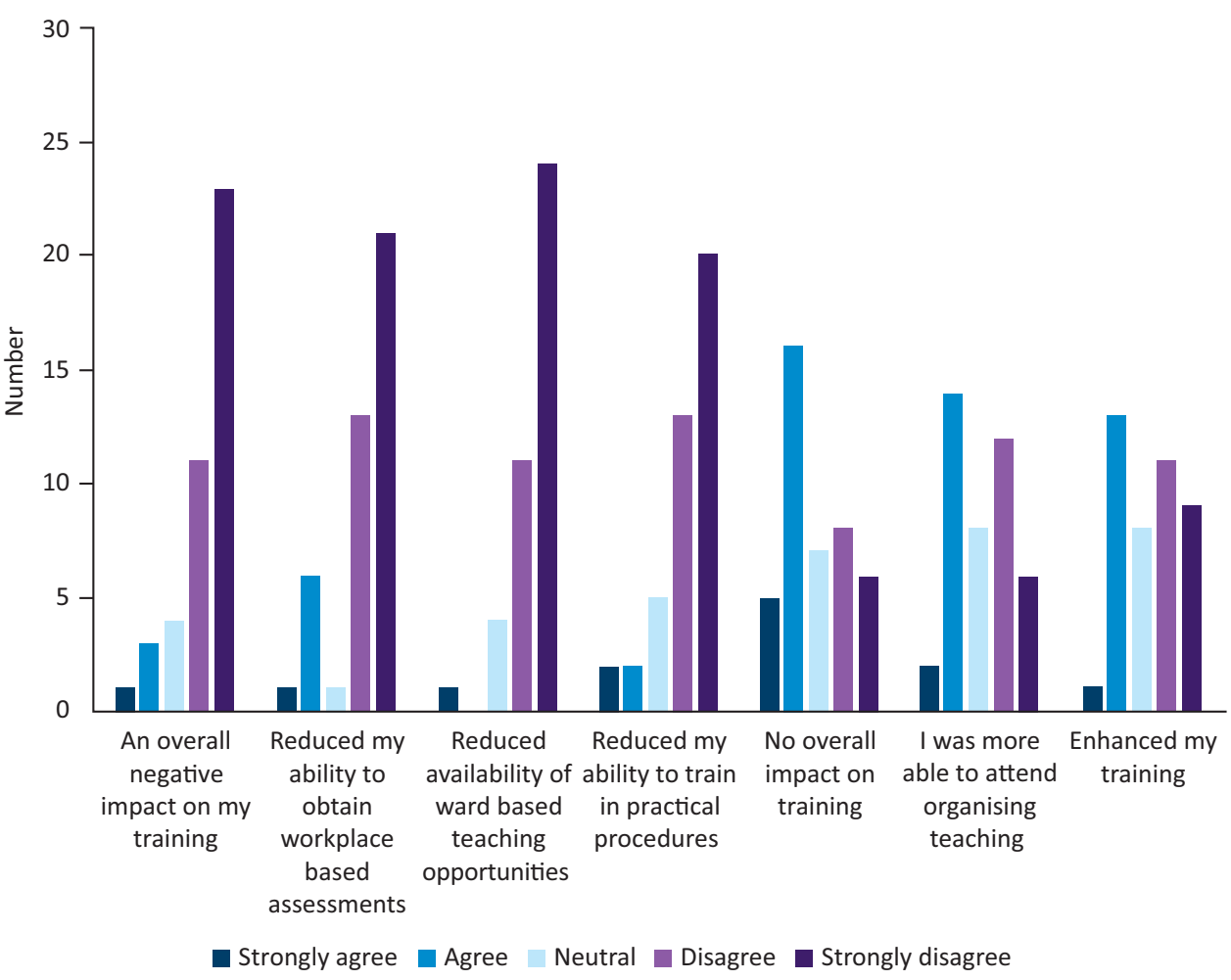

Fig 1. Junior doctor responses to statements regarding the impact on education and training of the introduction of PAs to their workplace. four $(9.6 \%)$ junior doctors felt that the presence of the PA reduced access to practical skills training, 33 (79\%) reported no reduction. Evidence from focus groups suggested that in some situations, PAs were facilitating acquisition of procedural competencies; F2 (2):

For the [core] trainees, when lumbar punctures came in to do ... they would go and do them and the PA would stay and do the bloods somewhere else or carry on clerking, so it would free up an opportunity for them to learn how to do a procedure.

Survey responses regarding informal learning opportunities are shown in Fig 1. Many junior doctors described specific instances where training had been enhanced by PAs facilitating informal training opportunities; ST (1):

When you're doing ward round and the nurse comes over and says, 'so and so needs a cannula now ... you need to do it now' and the consultant's doing the ward round ... the PA will go off and do the cannula and leave the F1s on the ward round. Yes, they might think it's just a ward round but actually they are learning something because the consultant's there explaining.

All participants were specifically asked about observed examples of training opportunities being diverted to PAs. One focus group participant had observed diversion of a potential training opportunity; F2 (3):

What I saw was that the PAs went on the ward round with the consultant and got bedside teaching but the F1s were left doing the to take outs. I think it should be the other way around because you can learn a lot on the ward round with the consultant.
Twelve survey respondents (20.7\%) reported that some training opportunities for junior doctors were being diverted to PAs. One such incident was described by a survey participant; ST (survey):

I was sat writing drug charts and ordering investigations that the PA couldn't order, whilst the PA discussed patients with the consultant.

In total, five junior doctors (11.9\%) and no consultants felt that the presence of the PA had an overall negative effect on medical training.

\section{Concerns for future potential impact of PAs on training}

Although very few specific examples of training opportunities being diverted were elicited, participants reported ongoing concerns about the potential impact on training even after the PAs had started work. Data suggested that experience of working with PAs may have reduced concerns about negative impact on training. Nine (39.1\%) doctors in survey 1 felt that training opportunities would be diverted to PAs compared to 12 (20.7\%) respondents to survey 2 reporting this having happened in practice. Concern regarding potential situations where training opportunities may be diverted were commonplace; F1 (1):

If you're in a specialty that means clerking in patients, there probably would be some element of competition if you could see that they were taking some of your clerking away, that would probably be detracting from the ... learning experience.

Ongoing concern about undetected impact on training opportunities was evident in many responses with responses 
Fig 2. Having worked with a PA, do you feel differently about the role? Combined results for all doctors responding to survey 2 .

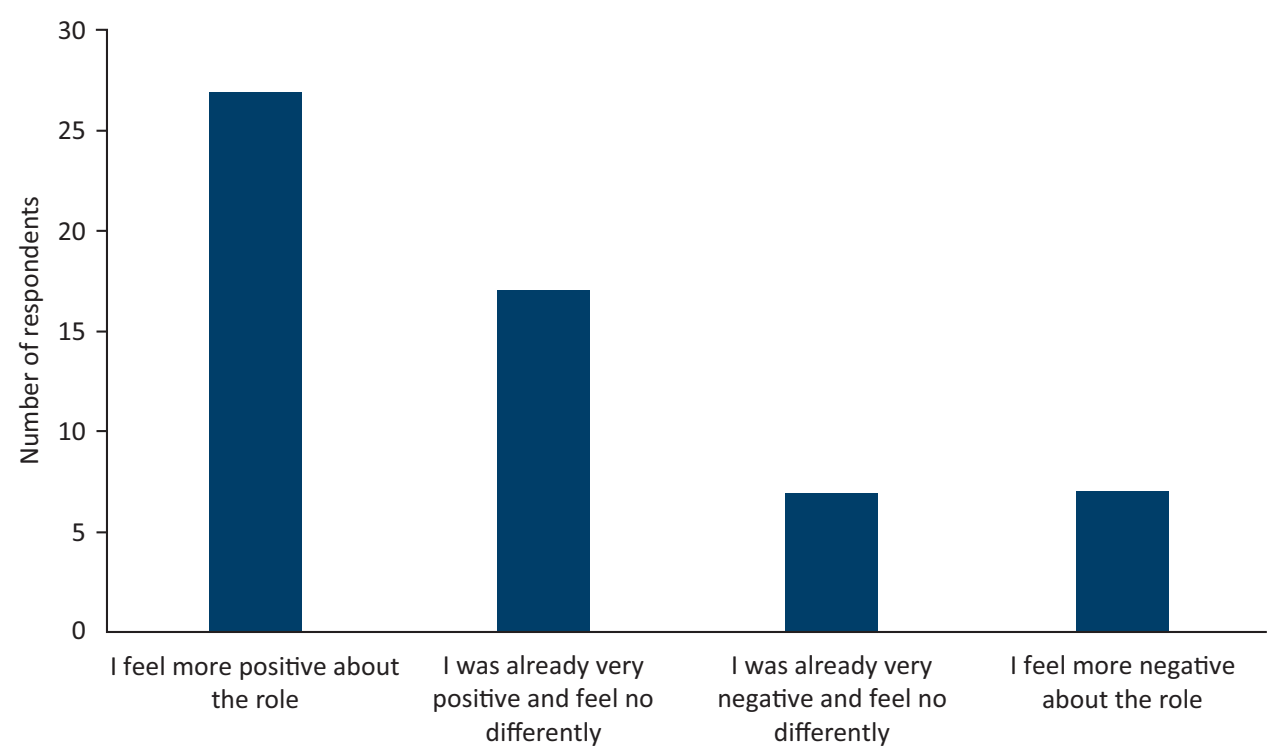

indicating that this may have emerged from external sources; CT (1):

It's just that social media argument of, 'Are they taking away our learning opportunities?' In reality, no, I don't think they are at all, but there's just that occasional feeling, maybe?

\section{Training needs of PAs}

Uncertainty about the educational needs of NQPAs emerged from focus groups as a key issue influencing participants' concerns about potential future impact on PGME. Whilst structured career progression for PAs is not yet clearly defined, NQPAs self-identified throughout the study as learners; PA (8):

I think that if you're at the point in your career where you don't want to learn anything new and you don't want to try anything new, it's probably time for you to give up.

Junior doctors however were often uncertain how the position of the PAs related to training and career progression; F1 (2):

They're qualified but are they in training? Are they in training? Or have they popped out of university fully formed?

As such, many junior doctors were unclear as to the PA education and training needs and the extent to which these overlapped with those of postgraduate medical trainees; ST (2):

\section{Do I teach them; do I not teach them?}

\section{Overall Impression of PAs}

All doctors participating in survey 2 were asked about how working with a PA had influenced their overall perceptions of the role. $44(75.9 \%)$ felt their experience had left them positive, or more positive about PAs. Results are summarised in Fig 2.

\section{Discussion}

This study has found that the introduction of PAs within five hospitals in northern England was reported to have no overall detrimental effect on PGME. In many cases, the PAs were perceived as having potential to enhance postgraduate medical training.

Introduction of new roles has been previously shown to cause concern about role overlap and impact on training from existing professional groups. ${ }^{24}$ As such, it is no surprise that this has been the case with PAs. The findings reported here support previous research indicating that most doctors are satisfied with the role of PAs with whom they work. ${ }^{25,26}$ Specific concerns about PAs having an overall negative impact on training failed to materialise with results indicating that overall PAs did not detract from PGME with significant potential for PAs to facilitate PGME. Participants were able to describe specific examples of PAs contributing indirectly to training of junior doctors, predominantly by releasing them from service provision to access formal and informal training opportunities. This supports previous assertions and international experience that both ACPs and PAs have potential to positively impact medical training. ${ }^{17,19,26,27}$

There was a disparity between survey and focus group participants regarding observed diversion of training opportunities to PAs. A greater proportion of survey respondents reported that they had observed training opportunities for junior doctors being taken by PAs although only one such instance was explicitly described in the survey. Both instances of training opportunities being diverted to PAs elicited in the study involved PAs being observed to benefit from an informal learning opportunity whilst a junior doctor engaged in service provision. This is an area that warrants further exploration in future work. Participants, particularly junior doctors, reported ongoing apprehension regarding undetectable or future negative impact of PAs on the participants' training even if they had not observed any diversion of educational opportunities. Whilst some of these concerns were acknowledged to have emerged from social media rather than personal experience, there remained a sense of unease about fully embracing the PA as a facilitator of medical training. This may well result from participants' uncertainty about current and ongoing education needs of the NQPAs, therefore to what extent there may be future competition for learning opportunities. 


\section{Strengths}

Our study has several strengths. The findings emerged from participant experience in a clinical setting across a number of hospitals working across different specialties therefore represent a broad range of exposure to working with different PAs. Participants were recruited from a variety of levels of seniority and backgrounds. The mixed methods enabled appreciation of both breadth and depth of understanding of participant experience. Experiences relating to all NQPAs working in secondary care within the region were represented.

\section{Limitations}

Our selection for invitation to surveys was based on a maximal variation purposive sampling technique, aiming to recruit three colleagues to surveys for each PA working within the organisation. Whilst this may risk sampling bias, we also invited all junior doctors to participate in the focus groups and interviews, thereby mitigating this risk. Response rate to survey 1 was low, however, results presented emerged from focus groups or survey 2 , which achieved a response rate of $40.7 \%$, in line with expected response rates for similar studies. ${ }^{28}$ Survey responses and focus groups may have been skewed by self-selection bias, as colleagues with a particularly positive or negative experience may be most motivated to participate. However, we consider that as the aim of the study was to explore experiences of working with PAs, appreciating both negative and positive responses is informative and useful.

This study was not designed to provide an objective, quantifiable assessment of PAs' impact on PGME. The aim was to understand participant experience and perceptions of the impact of PAs on PGME and the results represent the opinions and observations of participants, which are inherently subjective.

The findings presented relate to the impact of a cohort of 20 PAs working across five hospital sites - the majority were NQPAs and most were employed in 4-month rotational posts. Although there is little published data on the nature of NQPA posts, our experience is that most PAs in the UK do not rotate through different specialties. We suggest that NQPAs require more training than experienced PAs and that rotating through different specialities necessitates additional training compared to permanent positions. Therefore the training requirements of PAs in this study are likely to be greater than the majority of PAs in the UK and this study may over-estimate PA training needs and competition for training opportunities, and may under-estimate the potential for PAs to contribute to PGME compared to the national perspective.

\section{Implications}

The work presented here has implications for several areas. At a local level, it appears that PAs have no overall detrimental effect on PGME and have the potential to positively impact training. Widespread concerns about a deterioration in the availability and quality of training opportunities for junior doctors failed to emerge - this will be reassuring to organisations planning to introduce PAs to their workforce. A positive departmental attitude to education promoted inter-professional collaboration and integration. In areas of particularly intense workload, junior doctors acknowledged organisational efforts to address their training concerns by employing PAs. By promoting PA role awareness prior to employment, employing organisations may be able to prepare their existing workforce and plan how PAs can positively impact PGME and minimise any detrimental effects such as diversion of informal training opportunities.

At a policy level, the work presented here supports the assertion that new roles can potentially support and facilitate medical education. ${ }^{14,29}$ The number of UK qualified PAs is set to triple by 2020 . As such, the findings of this study with regards to ongoing availability of training opportunities for are reassuring for training bodies such as Health Education England and the royal colleges. ${ }^{6}$ Finally, it is clear that further research is needed the findings presented relate to a small cohort of NQPAs across different sites. Whether these are representative of PAs working at scale will need further investigation. In addition, further work is required to assess quantifiable indicators of training quality and availability such as attendance at teaching, postgraduate examination results, training survey feedback and exception reports.

In summary, we report that views of the impact of PAs on PGME are mixed with no overall detrimental effect. Participants experienced initial concerns but in practice, the introduction of a cohort of NQPAs was reported to have some positive impact on training, relieve pressure on existing staff and facilitate release from service provision to undertake learning opportunities.

\section{Ethics}

Written consent was gained from all participants. Ethical approval was granted by the University of Leeds School of Medicine Research and Ethics Committee. Research is reported in line with the consolidated criteria for reporting qualitative research.

\section{Supplementary material}

Additional supplementary material may be found in the online version of this article at www.clinmed.rcpjournal.org:

S1 - survey questions and responses relating to PA impact on PGME.

\section{References}

1 Royal College of Physicians. Focus on physicians. Census of consultant physicians and higher specialty trainees 2017-18. London: RCP, 2018.

2 General Medical Council. National training surveys 2018: Initial findings report. London: GMC, 2018.

3 Tasker F, Newbery N, Burr B, Goddard AF. Survey of core medical trainees in the UK 2013 - inconsistencies in training experience and competing with service demands. Clin Med (Lond) 2014;14:149-56.

4 NHS Providers. There for us. A better future for the NHS workforce. London: NHS Providers, 2017.

5 Royal College of Physicians. Guidance on safe medical staffing. Report of a working party. London: RCP, 2018.

6 Ritsema T. Faculty of Physician Associates Census 2017. London: RCP, 2017.

7 Aiello M, Roberts KA. Development of the UK physician associate profession. JAAPA 2017;30:1-8.

8 Health Education England. Multi-professional framework for advanced clinical practice in England. HEE, 2017.

9 Royal College of Physicians. Who are physician associates? London: RCP, 2017.

10 Armitage M. Advanced-care practitioners - friend or foe? Lancet 2006;367:375-7. 
11 Hardy T, Dovell G, Hindle M, Bateman K, Desmond A. Physician associates - junior doctors' perceptions ahead of deployment. Int J Surg 2017;47:S69.

12 Rimmer A. Will physician associates be replacing doctors? BMJ 2014:349:g5439.

13 Physician associates - a very mixed experience. www.bma.org. uk/connecting-doctors/b/work/posts/physician-associates-a-verymixed-experience [Accessed 17 July, 2018].

14 Royal College of Surgeons. A question of balance. The extended surgical team. London: RCS, 2016.

15 Matthews-King A. GP training cuts necessary to allow HEE to 'develop physician associates'. Pulse 2017.

16 Goudie S. The physician assistant. BMJ 2010;340:c2462.

17 Ross N, Parle J, Begg P, Kuhns D. The case for the physician assistant. Clin Med (Lond) 2012;12:200-6.

18 Dacre J, Watkins J. The RCP and FPA's response to Pulse article on Health Education England's physician associate training support. London: RCP; 2017.

19 Stahlfeld KR, Robinson JM, Burton EC. What do physician extenders in a general surgery residency really do? J Surg Educ 2008;65:354-8.

20 Roberts S, Howarth S, Millott H, Stroud L. "What can you do then?" Integrating new roles into healthcare teams: regional experience with physician associates. (unpublished data).

21 Corbin JM, Strauss A. Grounded theory research: procedures, canons, and evaluative criteria. Qual Sociol 1990;13:3-21.

22 Plowright $\mathrm{D}$. To what extent do postgraduate students understand the principles of mixed methods in educational research? International Journal of Multiple Research Approaches 2013;7:66-82.
23 Braun V, Clarke V. Using thematic analysis in psychology. Qualitative Research in Psychology 2006:3:77-101.

24 Cummings GG, Fraser K, Tarlier DS. Implementing advanced nurse practitioner roles in acute care: an evaluation of organizational change. J Nurs Adm2003;33:139-45.

25 Williams LE, Ritsema TS. Satisfaction of doctors with the role of physician associates. Clin Med (Lond) 2014;14:113-6.

26 Farmer ], Currie M, West C, Hyman J, Arnott N. Evaluation of physician assistants to NHS Scotland. Inverness: Centre for Rural Health, 2009.

27 McDonnell A, Goodwin E, Kennedy F, Hawley K, Gerrish K, Smith C. An evaluation of the implementation of advanced nurse practitioner roles in an acute hospital setting. J Adv Nurs 2015;71:789-99.

28 Cunningham CT, Quan H, Hemmelgarn B et al. Exploring physician specialist response rates to web-based surveys. BMC Med Res Methodol 2015;15:32.

29 Forbes-Pyman R, Woosey D. Pioneering the role of physician associate: the value of education and peer support. London: RCP, 2017.

Address for correspondence: Dr Sam Roberts, Leeds Institute of Health Sciences, University of Leeds, Worsley Building, Clarendon Way, Leeds, LS2 9JT, UK.

Email:sam.roberts8@nhs.net

\section{Royal College of Physicians}

\section{Simples and rarities suitable and honourable to the college}

The ninth volume in the RCP500 series of 10 books is set in the context of manuscript and book production. This volume describes the Dorchester Library and how its rich and diverse collection illustrates the history of medicine and ideas.

\section{Order by phone or online}

Tel +44(0)20 30751358 or visit www.rcplondon.ac.uk/shop

Price: $£ 12$ (Postage and packaging not included) $10 \%$ discount for fellows and members,

$15 \%$ discount for foundation doctors and students

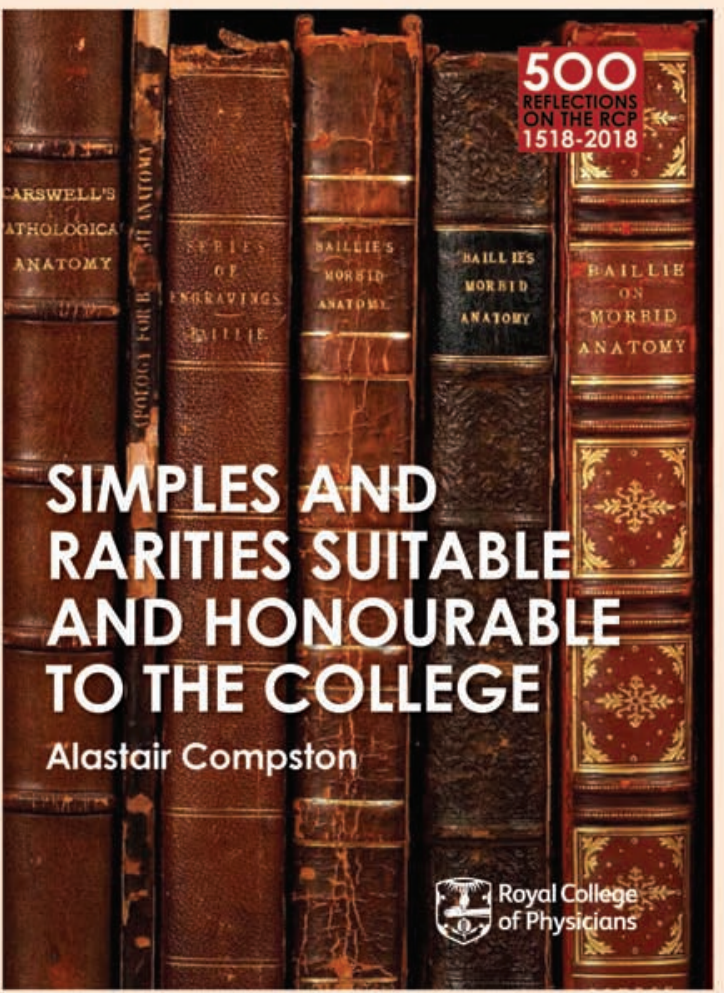

